\title{
Biological weapons and health protection against biological terrorism
}

\author{
Marta Blahova* \\ Tomas Bata University in Zlín, Nad Stráněmi 4511, 76005 Zlín, Czech Republic
}

\begin{abstract}
The possibility of using biological means has become a reality. A biological attack is the deliberate use of living microorganisms or their toxic products to cause disease and death in humans, animals and plants. Methods of biological attack and its main characteristics. Protection against biological attack and its basic articles. Possibilities of protection against biological attack and division of protective measures. Preventive measures and measures for rapid disease control.
\end{abstract}

\section{Introduction}

The possibility of using biological means has become a reality. The possibility of using them and the target that can be directly or indirectly targeted, including a terrorist attack, determines that defense and protection against them must primarily be a matter for those components in the state whose job is similar peace issues and military specially prepared bodies. The article summarizes the existing knowledge of biological weapons, shows their problems and gives an outline of how to carry out defense and protection against them, acquaints them with the basic issues of epidemiology in particular and should be the starting point for further study.

\section{Brief history of biological attacks}

A biological attack is the deliberate use of living microorganisms or their toxic products (toxins) to cause disease and death in humans, animals and plants. But the goal of biological attack is always human. It may be threatened by illness or death, directly or indirectly, by attacking its nutrition. The experience that until recently, every war has been linked to the occurrence of multiple epidemics and infections and that they have generally caused greater losses than the armed conflict itself, has already led to attempts to infect the enemy lines in the Middle Ages. However, in the current development of bacteriology, the possibility of using a biological weapon in a somewhat different dimension, on a scientific basis at a given time and level of knowledge [1], is being considered.In 1915,
German agent Gregersen brought a plague culture to Archangel, via the US, in order to provoke a plague in St. Petersburg. A year later another German agent was caught in Russia with a detailed plan of infestation of water pipes. In 1916, a shipment addressed to the local German embassy was captured in Romania, containing ampoules of anthrax and glanders. They were accompanied by a written instruction clearly demonstrating that these cultures should be used to diversify and spread these diseases among animals. The investigation of this incident was carried out by the famous Romanian microbiologist Babes.

In 1917, agents who were trying to spread glanders in French cavalry horses were repeatedly caught on the French Western Front. Obviously, only the rapid end of World War I prevented the then command of the imperial army from using a biological weapon on a much wider scale.These and other experiences gave rise in 1925 to a meeting in Geneva to ban bacteriological warfare. And it is very remarkable that all states except Japan signed this treat and Germany. The Geneva Protocol was preceded in 1922 by the Washington Conference, which was very skeptical of the biological war.The debate on bacteriological war was revived in the years before World War II. At the time many experts have expressed doubts about the possibility of using a bacteriological weapon. However, recently, microbiological techniques have advanced so much that the possibility of producing highly effective disease agents not only in terms of quantity but especially in the diversity of species.

Chemical, nuclear and biological weapons are collectively considered weapons of mass destruction because they can cause mass disease and death. In addition to military targets, they are primarily threatening the civilian population [2]. 
A biological weapon differs by causing disease and death by reproduction disease-causing organisms in the infected organism of humans, animals or plants. However, if a bacterial toxin is used to attack, the effect is similar to that of a chemical weapon. Until recently, the use of a biological weapon was limited to a number of pathogenic bacteria, viruses, rickets, or pathogenic fungi commonly found in the world with known properties. The fundamental mistake was to think that the number and choice of biological agents is fundamentally limited. Unfortunately, the expansion of microbiology and virology allows not only a significant influence on the virulence of the various agents of the disease, but the deliberately induced changes in their antigenic structure, resistance to antibiotics in the genetic structure and other properties only increases the potential danger of using biological agents. Therefore, the issue of protection against biological attack is now seen as an extremely responsible and topical task of the health service of each state [2].

Problems arising from ensuring effective defense against biological weapons are, like chemical and radiological weapons, extremely complicated. Yet, in the protection against biological agents, there is an undeniable and far-reaching advantage - the target of attack by a biological agent is a human (plant, animal) in which an infectious disease or intoxication is to be induced. Protection means early detection, identification, prophylaxis, treatment or administration of drugs [2].

Biological attack promoters often refer to the fact that the preparation and use of biological resources is disproportionately cheaper than the use of chemical or nuclear weapons. At the same time, we must realize that any attack by biological means always counts on causing panic and fear. Here is the main role of preparedness of the anti-epidemic components of the state [2].

\section{Methods of biological attack}

Infectious aerosols are particularly dangerous for humans and animals. Much of the experimental work has proven to be an airborne infection in some diseases such as Q-rikettsiosis or tularemia is sufficient to inhale ten microorganisms. Furthermore, it has been shown that the incubation period is shortened at high infectious doses. Nowadays, it is no longer a technical problem to hit an aerosol containing a large concentration of microorganisms. A number of experimental works using fluorescent particles or nonpathogenic bacteria have shown this.

In particular, micro-organisms are:

- viruses (smallpox agents, ornithoses, psittacosis, influenza, inflammation of the brain, yellow fever, dengue, rabies) rickettsia (Q-rikettsiosis)
- bacterial agents of the disease (P. pestis, P. tularensis, M. mellei, M. pseudomallei, brucela, B. anthracis.)

- pathogenic fungi (Histoplasma capsulatum, Coccidioides immitis, Nocardia

- $\quad$ and Blastomycers.)

- $\quad$ bacterial toxins (practically unlikely).

The second very dangerous method of biological attack is the spread of infections or intoxications by the alimentary route [3].

\section{Main characteristics of biological attack}

On today's level of knowledge and technical capabilities, it can be assumed that today there is no problem preparing a large number of pathogenic microbes, viruses, fungi and toxins that can be used to attack. Using modern cultivation methods for bacteria (flowing environments) and viruses (tissue cultures) it is not difficult to propagate cultures in virtually any amount and maintain them for a long time virulent, respectively. toxic (frozen, lyophilized) [3].

The attack can be done openly (in case of a war conflict) or covertly (a terrorist attack). Terrorist attacks can be carried out in many places simultaneously. If they are directed at water resources, they can result in a large number of people. Of course, terrorist attacks can also be conducted against individuals and small groups of key people. It can be assumed that the attacker will always try to make it more difficult to recognize the disease, as well as the possibilities of specific treatment and prevention. It can be assumed that it will use two or more disease agents to attack, especially in the case of air attack, infestation of water resources (alimentary route).

\section{Biological attack can be used: \\ - bacteria, \\ - rickettsie, \\ - viruses, \\ - mushrooms, \\ - bacterial toxins.}

In the order of danger, viruses, which are also the most difficult to defend, are in the first place. Conversely, highly virulent bacterial infections, once so feared as plague, cholera, have now virtually lost their dangers. It is very difficult to consider which resources an attacker chooses. A hidden attack can be conducted long before the opening of open hostility. The means of choice and the route of attack will depend on the relative resistance and stability of the microorganism used or poison under the conditions, on the ease or difficulty of detection, sanitation (disinfection or decontamination). This is connected with the possibility of timely securing of specific treatment and especially prevention in the affected 
area. In general, it can be assumed that a biological attack against a highly developed country, with a wellorganized sanitary and health service, counts primarily on the effect of single or repeated exposure than with the possibility of spreading such an infection in the population [3].

It is believed that such use of a biological agent will immediately lead to measures that substantially reduce the spread of the disease. Very easily vulnerable water supply is particularly vulnerable. For comparison:

1. Less than $5 \mathrm{~kg}$ of purified lyophilized botulinum toxin in 5 million liters of water induces botulism in more than $90 \%$ of the exposed persons.

2. In order to cause fatal poisoning after drinking $100 \mathrm{ml}$ of poisoned water, $10,000 \mathrm{~kg}$ of potassium cyanide would be required for a water tank with 5 million liters of water.

Similarly to drinking water supply, common eating, food production can be subject to attack. It is important that there is no need for any large special equipment or great expertise to prepare the required amount of bacterial cultures The disease-related attack by the alimentary route is predominantly represented by Salmonella, Shigella, Brucella, V.cholerae, P.tularensis. However, bacterial toxins (various types of botulinum toxins, tetanus toxin, Sh.disenteric toxin, staphylococcal enterotoxin, etc.) are particularly dangerous. Of the viral infections, enteroviruses (poliomyelitis viruses, ECHO, coxsackie), arboviruses / encephalitis viruses, ricketsia (C.burneti) may be used. Finally, the third method of biological attack is the use of infected arthropods. For the sake of completeness, it is necessary to mention the fourth group in the epidemiological classification of diseases, that is the deliberate spread of disease agents, where the entrance gate is broken skin and mucous membranes (especially anthrax).

In addition to the enumerated, commonly known and most important diseases (or groups) that may be considered, the possibility of using unknown pathogens (isolated in remote parts of the world) or new antigen types and properties may or may not be considered. These may be deliberately prepared antigenic mutants, with deliberately prepared resistance to known and commonly used drugs, or a combination of two more disease agents. The indisputable advantage of biological agents is the fact that the success of the attack takes effect only after a certain latency period. That the first signs of the disease are not always typical, not to mention that the deliberate use of a combination of multiple disease agents together can cause atypical manifestations of the disease. When we consider how difficult and long-lasting isolation is in many diseases, especially viral, then the protection against biological agents is delayed. Of course, you increase the undesirable psychological effect of the attack.
The basis of protection against biological biological agents is perfectly materiál and staffed hygienicepidemiological service, especially microbiological and virological laboratories, availability of immunological materials, refinement of laboratory and diagnostic procedures [4].

\section{Protection against biological attack}

The biological attack is essentially a perverted misuse of all knowledge of the nature, causes, distribution, suppression and prevention of naturally occurring infections and poisonings. Therefore, protection against biological attack is not a completely new problem. It is necessary to adapt our knowledge and possibilities of protection against this new situation, to strengthen and supplement it materially and personally. The difference must be only in the effort and scope of the means used, as the nature of the thing requires.

The basic element of protection is correct and rapid diagnosis of infections and intoxications. This knowledge also includes prophylaxis of other diseases, their treatment, specific prevention, sanitation, etc.

Considerable attention is paid to non-specific detection issues. Systems are being developed that rely on either the determination of protein content in air, or the measurement of atmospheric particle size, and the like.

Therefore, the protection against biological attack also presupposes a precise knowledge of all the diseases that occur in animals and plants. Immunological studies also contribute to this. Essentially rapid detection and identification of biological agents is incomparably more difficult and complex than detecting radiological or chemical attack

\section{Possibilities of protection against biological attack}

Protective measures against biological attack can be divided into groups:

a) detection,

b) reporting and general (preventive) antiepidemic measures;

c) personal protection

d) collective protection;

e) decontamination and decontamination;

f) causal treatment,

g) defense against psychological attacks and panic.

Biological attack attack detection is not an easy matter. The attack by biological means is fundamentally different from attacks by other weapons

Corresponding author:m6_blahova@utb.cz 
of mass destruction, because its action is hidden for many hours (for bacterial toxins), for days or weeks, depending on how long the incubation period for the individual diseases and respiratory diseases is. otrav. This is the trickery and other danger of a biological attack. Only the result of a biological attack, the occurrence of the disease can be the first signal of a biological attack. The intelligence service undoubtedly plays an important role in ensuring protection against biological attack. It provides information not only about what event. biological attack agents are ready to use, but also indirect indicators from which judgment can be drawn

about possible preparations for a biological attack. However, protection cannot be limited to diagnostic work. When a biological attack is suspected, it is necessary to use all the means for individual and collective protection and decontamination affected [4].

Persons and animals in the affected area must be examined and monitored for at least 21 days and quarantined. If some indicators point to a particular dangerous disease against which specific prophylaxis is possible, the affected persons can be protected. If a particular causative agent of the disease in which active immunization can be found has to be performed. It is understandable that immunization of all populations cannot be pre-performed by all the means at our disposal, especially since many immunizations have only a short-term effect. The most important of all is good readiness, readiness and a high level of serum production and vaccines, including those that do not normally occur in the area [4].

Collective protection means that staying in confined spaces, taking advantage of the protective properties of buildings, constitutes a substantially reduced exposure to infection. Some biological attack agents may contaminate the soil, the exterior and interior walls of objects, various exposed objects and endanger them for a short or longer period. Inexpensive easy-to-use disinfectants can be used for decontamination. Complex and the challenge is only to ensure sufficient supplies and the possibility of producing decontamination agents. Depending on the extent of the biological attack, it is necessary to think about the hospitalization of all the sick, that it will be necessary to calculate with some improvisation. But what must always be secured is the means of specific and causal treatment. Unfortunately, we also have to assume that human disease will be the first sign of a biological attack. Preparing against biological attacks must assume certain supplies and production of sera, chemotherapeutics, antibiotics, corresponding to those diseases that are exotic to us or rarely occur.The treatment of diseases caused by biological attack, in comparison with the treatment of naturally occurring diseases, represents mainly a quantitative-qualitative difference. Unfortunately, there are no specific treatments available for most viral infections.

In particular, the following are required for antiepidemic measures:
- accelerated epidemiological examination to determine the cause of the disease (epidemic)

- $\quad$ securing isolation and quarantine

- ensuring mass vaccination of vulnerable populations

- disinfection, disinsection, rodent control.

In terms of hygiene measures:

- $\quad$ ensure the supply of drinking water,

- ensure food supply,

- perform wastewater control and remove impurities,

- control of insects and rodents.

The system of anti-epidemic measures is focused both on preventive measures and on the suppression of already existing diseases [5].

Preventive measures include, in particular, the increased risk of infections resulting from the accumulation of people, from shared accommodation and eating in poorly or unsatisfactory conditions. Naturally, protective vaccination is of great importance in protecting against biological attack. However, it is absolutely impossible to protect the population against all diseases, their use in biological attack is possible. It is much easier to use vaccines that induce long-term and solid immunity than those that are short-lived and very relative.

The basic immunization schedule remains vaccinated against:

$\begin{array}{ll}- & \text { smallpox } \\ \text { - } & \text { tetanus } \\ \text { - } & \text { typhoid fever and paratyphus } \\ \text { - } & \text { botulism (A-E) } \\ \text { - } & \text { poliomyelitis } \\ \text { - } & \text { tularemia } \\ \text { - } & \text { encephalitis } \\ \text { - } & \text { brucellosis } \\ \text { - } & \text { plague } \\ - & \text { yellow fever and some other diseases. }\end{array}$

Measures for the rapid suppression of existing diseases. If a biological attack has been suspected, a detailed epidemiological examination should be provided if the use of the biological agent is proven or justified:

a) promptly identify or estimate the extent of the attack and mark the affected area;

b) actively seek, isolate, suspect and treat illnesses or diseases,

c) ensure decontamination, disinfection and / or rodent control

d) take further measures to prevent the further spread of the disease.

Biological agents, with their unusual, insidious and delayed manifestations of attack, are particularly well suited to their undesirable psychological effect and panic [6]. 


\section{Discussion}

The aim of the article was to acquaint readers with the issue of Biological Weapons and Health Protection against Biological Terrorism. We briefly discussed the history of biological attacks. Furthermore, we have further explored the main characteristics of the biological attack and, in the main part, devoted ourselves to protection and protection against biological attacks, which occupies the main part of the article and is given adequate space. The importance of protection against biological attacks is crucial to the issue addressed by this article. In the last part, we looked at measures to quickly suppress the emerging diseases and their benefits and benefits in practice.

Acknowledgments: This research was based on the support of the Internal Grant Agency of Tomas Bata University in Zlín, the IGA / FAI / 2019/003 project and the Institute of Safety Engineering, Faculty of Applied Informatics.

\section{References}

1. I., MAŠEK, M., ZEMAN,.: PotSoPW (2003)

2. A., GAVEL, : Aoovzbh. (2012)

3. H., JANSEN,: BWbaB. (2014)

4. I., MAŠEK, M., ZEMAN,: PoueitoFB. (2004)

5. P., MATES,: PpPpsK. (2004)

6. M., Šenovský,: PotP (2005). 